\title{
Olive Oil Quality and Sensory Changes During House-Use Simulation and Temporal Assessment Using an Electronic Tongue
}

\author{
Nuno Rodrigues ${ }^{1,2} \cdot$ Letícia Oliveira $^{1,3} \cdot$ Lorena Mendanha $^{1,3} \cdot$ Mohamed Sebti ${ }^{1,4} \cdot$ Luís G. Dias $^{1}$. \\ Souheib Oueslati $^{4} \cdot$ Ana C. A. Veloso $^{5,6} \cdot$ José A. Pereira $^{1} \cdot$ António M. Peres $^{1,7}$ (I)
}

Received: 9 February 2018 / Revised: 18 May 2018 / Accepted: 18 May 2018

(C) 2018 AOCS

\begin{abstract}
During domestic usage, olive oil bottle manipulation may lead to a quality decrease due to agitation and oxygenation. Therefore, assessing the domestic consumption time period during which the initial quality grade is retained may allow including this information as a recommendation, ensuring olive oil consumers' satisfaction. Temporal changes of physicochemical, chemical, and sensory parameters of extra-virgin olive oils (EVOO) were monitored during 1-month simulated house-use conditions. It was observed that $K_{232}(R$-Pearson $\geq+0.81)$ and $\Delta K$ increased resulting in a significant olive oil quality decrease from EVOO (during the initial 21 days of simulated usage) to lampante olive oil (after 28 days of simulated usage) as
\end{abstract}

António M. Peres

peres@ipb.pt

1 Centro de Investigação de Montanha (CIMO), ESA, Instituto Politécnico de Bragança, Campus Santa Apolónia, 5300-253, Bragança, Portugal

2 Departamento de Ingeniería Agrária, Universidad de Léon, Av. Portugal, $n^{\circ} 41,24071$, Léon, Spain

3 Instituto Federal de Educação Ciência e Tecnologia Fluminense, Bom Jesus do Itabapoana, 28360-000, Rio de Janeiro, Brazil

4 Laboratoire Materiaux, Molécules et Applications (LMMA), Institut Préparatoire aux Etudes Scientifiques et Techniques (IPEST), BP 51, 2070, La Marsa, Tunisia

5 Instituto Politécnico de Coimbra, ISEC, Departamento de Engenharia Química e Biológica (DEQB), Rua Pedro Nunes, Quinta da Nora, 3030-199, Coimbra, Portugal

6 CEB-Centre of Biological Engineering, University of Minho, Campus de Gualtar, 4710-057, Braga, Portugal

7 Laboratory of Separation and Reaction Engineering-Laboratory of Catalysis and Materials (LSRE-LCM), ESA, Instituto Politécnico de Bragança, Campus Santa Apolónia, 5300-253, Bragança, Portugal well as the appearance of rancid sensation. As lampante olive oils cannot be commercialized, it is pertinent to establish olive oil shelf life under usual home-use conditions. Principal component analysis allowed grouping the olive oils according to home-use time period and how bottles are stored after their first opening, showing that the overall olive oil physicochemical and sensory characteristics changed with the domestic-use time period. Finally, a potentiometric electronic tongue coupled with linear discriminant analysis was used to discriminate olive oils according to the domestic-use time period (leave-one-out crossvalidation sensitivities $\geq 95 \%$ ). Thus, this device could be used to indirectly assess the quality of the remaining bottled olive oil by establishing for how long an olive oil bottle has been used under domestic conditions.

Keywords Olive oil · Physicochemical analysis · Sensory analysis - Temporal changes - Electronic tongue

J Am Oil Chem Soc (2018).

\section{Introduction}

Recently, the study of olive oils has increased due to their applications in food, pharmaceutical, and cosmetic fields (Spatari, De Luca, Ioele, \& Ragno, 2017). Olive oil has unique sensory, nutritional, and health characteristics, making it highly appreciated by consumers. However, olive oil undergoes lipid oxidation and hydrolytic degradation during its storage along the distribution chain, which can dramatically change its final nutritional, health, and sensory properties, leading to the appearance of sensory defects like rancidity (Genovese, Caporaso, \& Sacchi, 2015), 
decreasing its final quality grade. The olive oil quality changes during the distribution/commercialization storage have been widely investigated under the conditions of different time periods, temperature ranges, lighting, and packing (Ayyad et al., 2015; Jabeur, Zribi, \& Bouaziz, 2016; Rodrigues, Dias, Veloso, Pereira, \& Peres, 2016; Salek et al., 2017; Sanaeifar, Jafari, \& Golmakani, 2018). In contrast, as recently pointed out by Genovese et al. (2015), researchers have mostly focused on the shelf life of the bottled olive oil during the product distribution rather than on the quality changes that may occur during domestic consumption. Indeed, to the best of our knowledge, only one study addressed the effects of home consumption conditions on the extra-virgin olive oil (EVOO) shelf life, the changes in free acidity (FA), peroxide values (PV), and phenolic compounds being monitored during 1-year storage at room temperature (Di Lecce, Bendini, Cerretani, Bonoli-Carbognin, \& Lercker, 2006). The domestic consumption of the bottled olive oil usually implies that after its first opening the headspace gradually increases during the house-use time period, until the full usage of the bottle. During this process, the olive oil is shaken due to the bottle manipulation, promoting the introduction of atmospheric oxygen into the bottle headspace at every use (Di Lecce et al., 2006; Genovese et al., 2015). This may lead to changes in the quality of olive oil such as the loss of positive-sensory attributes in the olive oil kept inside the open bottles (Genovese et al., 2015). Therefore, it would be helpful if it was possible to establish the shelf life of olive oil after its initial domestic use, i.e., during which time period the olive oil keeps its initial quality (EVOO and/or virgin olive oil, VOO) after the bottle is opened for the first time. This information, an estimate of the finest consumption time period after opening, could be then displayed by the olive oil producers in the bottle label, ensuring that under typical domestic-use conditions, the olive oil will keep the physicochemical and sensory characteristics that would allow it to retain its initial quality-grade classification (EVOO or VOO). This procedure would contribute to increase the consumers' confidence when buying this highvalue food product. In this study, the temporal changes of physiochemical, chemical, and sensory parameters of EVOO were measured, and their possible impact on the overall quality grade of olive oil was evaluated during 1-month house use (after the bottles being opened for the first time). During the simulated domestic use, olive oils were stored in bottles that remained open or were closed after each use and exposed to usual home temperatures and lighting conditions. Finally, the study aimed to verify if an electronic tongue together with linear discriminant analysis (LDA) could be used to discriminate olive oils according to the consumption time period $(0,7,14,21$, and 28 days after first opening of bottles), which could be further used as an indirect tool for assessing the quality of the remaining bottled olive oil after each domestic withdrawal. The use of potentiometric multisensor devices coupled with chemometric tools was previously reported. It aimed to qualitatively and/or quantitatively assess the physicochemical quality parameters, oxidative stability (OS), and positiveand negative-sensory attributes of olive oils, and, in some cases, to evaluate the effects of storage time and lighting conditions during the olive oil distribution and commercialization (i.e., unopened bottles) (Harzalli et al., 2018; Rodrigues et al., 2016; Veloso, Dias, Rodrigues, Pereira, \& Peres, 2016; Veloso et al., 2018).

\section{Materials and Methods}

\section{Olive Oil Samples}

Nine bottles of Arbequina EVOO (produced in the "Trásos-Montes" region of northeast Portugal during 2016/2017) were purchased from a local supermarket in Bragança (Portugal). According to the label information, the olive oil bottles were purchased 6 months after the packaging date. All olive oil bottles $(750 \mathrm{~mL}$ each) were from the same brand and lot and were stored in the original dark green glass bottles at the same temperatures (varying from 16 to $20{ }^{\circ} \mathrm{C}$ ) and under typical house-lighting conditions. Two bottles were left closed during the 1-month domestic-use simulation and the other seven bottles were first opened at day 0 , with the olive oil then analyzed every 7 days (at 0 , $7,14,21$, and 28 days). During the 1-month domestic-use simulation, $5 \mathrm{~mL}$ of olive oil was withdrawn daily from each of the seven opened bottles, plus $110 \mathrm{~mL}$ extra on the analysis days (every seventh day) for carrying out all physicochemical, chemical, sensory, and potentiometric assays. During each daily sampling, all bottles were smoothly shaken before the sample was withdrawn.

\section{Physicochemical, Chemical, and Sensory Analysis}

From each bottle, individual olive oil samples were withdrawn weekly (at $0,7,14,21$, and 28 days) and further subjected to physicochemical and sensory analysis, following the EU standard methods (EU No 1348/2013, 2013; EU No 61/2011, 2011). Five physicochemical quality parameters were evaluated: FA (in \% oleic acid), the PV (in meq $\mathrm{O}_{2} \mathrm{~kg}^{-1}$ ), and the specific coefficients of extinction at 232 and $270 \mathrm{~nm}\left(K_{232}, K_{270}\right.$ and $\left.\Delta K\right)$. In addition, the OS (in hours) of each olive oil sample was assessed using the Rancimat method (Rodrigues et al., 2016) and the total phenol (TP) content (in mg caffeic acid equivalents $\mathrm{kg}^{-1}$ oil) was determined according to Capannesi, Palchetti, Mascini, and Parenti (2000). For each olive oil sample, bottle storage type, and time period, all physicochemical 
assays were carried out in triplicate. Each sample was also evaluated by trained panelists from the olive oil sensory panel of the School of Agriculture of the Polytechnic Institute of Bragança following the recommendations of the International Olive Council (IOC, 2013, 2014). The intensity of the positive or negative attributes perceived was graded according to an intensity scale ranging from 0 (no sensory sensation perceived) to 10 (maximum intensity of the sensory sensation perceived). The quality grade (EVOO, VOO, or lampante olive oil, LOO) of each independent olive oil sample collected at different time periods $(0,7,14,21$ and 28 days) from each of the commercial olive oil bottles (closed or left open after each daily use) was set taking into account the physicochemical levels and the sensory mean intensities (EU No 1348/2013, 2013; EU No 61/2011, 2011; IOC, 2013, 2014): EVOO (FA $\leq 0.8 \%$ oleic acid, $P V \leq 20$ meq $\mathrm{O}_{2} \mathrm{~kg}^{-1}, K_{232} \leq 2.50$, $K_{270} \leq 0.22,|\Delta K| \leq 0.01$; ripe-fruit median intensity greater than 0 and median intensity of defects equal to 0 ); VOO (FA $\leq 2.0 \%$ oleic acid, PV $\leq 20$ meq $\mathrm{O}_{2} \mathrm{~kg}^{-1}$, $K_{232} \leq 2.60, K_{270} \leq 0.25,|\Delta K| \leq 0.01$; ripe-fruit median intensity greater than 0 and median intensity of defects greater than 0 and lower than 3); or LOO (in the other cases). All assays were performed at the laboratories of the School of Agriculture-Polytechnic Institute of Bragança.

\section{E-Tongue}

\section{E-Tongue Device and Set-Up}

The lab-made potentiometric E-tongue multisensor device comprised two print-screen sensor arrays, each containing 20 lipid polymeric sensor membranes (40 sensors in total), as previously described by Veloso et al. (2018) and Harzalli et al. (2018). The sensor membranes were a mixture of one lipid additive ( $3 \%$ : octadecylamine, oleyl alcohol, methyltrioctylammonium chloride, or oleic acid), one plasticizer ( 32\%: bis(1-butylpentyl) adipate, dibutyl sebacate, 2-nitrophenyl-octylether, tris(2-ethylhexyl)phosphate, or dioctyl phenylphosphonate), and polyvinyl chloride (PVC, $\sim 65 \%$ ) (Fluka, Madrid, Spain; minimum purity $\geq 97 \%$ ). Each sensor membrane was connected to a multiplexer Agilent Data Acquisition Switch Unit (model 34970A, Santa Clara, California, USA) controlled by the Agilent BenchLink Data Logger software (Santa Clara, California, USA) installed on a PC. Each potentiometric assay took $5 \mathrm{~min}$ and allowed recording the potentiometric signal profiles of the 40 sensor membranes generated through the establishment of electrostatic or hydrophobic interactions (Kobayashi et al., 2010). A reference $\mathrm{Ag} / \mathrm{AgCl}$ doublejunction glass electrode (Crison 5241, Barcelona, Spain) was used. The same sensor coding used in previous studies was adopted: Each sensor was identified with a letter S (for sensor) followed by the number of the array ( 1 or 2 ) and the number of the membrane (1-20, corresponding to different combinations of plasticizers and additives).

\section{E-Tongue Analysis: Olive Oil Sample Preparation and Potentiometric Assays}

All samples were electrochemically analyzed within the same day avoiding the need of statistical complex pretreatments of the signals to overcome the possible potential drifts (Veloso et al., 2018). For that, $10 \mathrm{~g}$ of olive oil was removed from each bottle after it was smoothly shaken, and then mixed with $100 \mathrm{~mL}$ of water-ethanol solutions (80:20, v/v) for 5-10 min using a vortex stirrer (LBX V05 series, LABBOX Labware, Barcelona, Spain) at $500 \mathrm{rpm}$. The mixture was left at ambient temperature for $60 \mathrm{~min}$, after which $40 \mathrm{~mL}$ of the supernatant solution was carefully withdrawn and immediately analyzed using the E-tongue. Electrochemical assays were performed in duplicate for each sample, with a third assay carried out if potentiometric signals recorded by each E-tongue sensor showed a coefficient of variation greater than $20 \%$ (values set according to the IOC regulations for sensory analysis).

\section{Statistical Analysis}

The existence of statistically significant main effects and/or of interaction effects of the bottle storage conditions after being first opened (closed or kept open after each use) and the domestic olive oil consumption time period $(0,7$, 14, 21, and 28 days) were evaluated using a two-way ANOVA. If a significant interaction effect was found, the analysis was firstly based on the interpretation of the estimated marginal mean plots for each parameter (physicochemical, chemical, or sensory) to determine the type of interaction (the additive or the nonadditive/disordinal effect for noncrossing or crossing lines). For the additive interaction effect, the main effects can be discussed separately (Student's $t$-test or one-way ANOVA followed, when appropriate, by the Tukey's post hoc multicomparison test). For the nonadditive interaction effect, the main effects cannot be interpreted individually (Field, 2009) and the evaluation should be restricted to the plots' trends. To further infer if the usual storage conditions of nonopened olive oil bottles did not have, per se, a significant effect on the observed temporal changes of the physicochemical and sensory parameters of the olive oils under the domestic consumption conditions, olive oils of the same commercial brand and lot, which remained in closed bottles during the same 28 days and in the same domestic environment, were analyzed and the data were compared by means of Student's $t$-test (assuming equal or unequal sample variances depending on the $F$-test) to those determined for the bottles opened at day 0 . Linear Pearson correlation coefficients $(R-$ 
Pearson) were also calculated to evaluate the possible existence of bivariate linear correlations between the timeperiod and the olive oils' physicochemical, chemical, or sensory parameters.

Principal component analysis (PCA) was used as an unsupervised pattern recognition technique with the objective of verifying if the physicochemical data fused with the sensory data would lead to a natural split of the olive oils according to the "bottle storage condition" and/or storage "time period" after each bottle had been opened for the first time. A low level of abstraction data fusion approach was applied, combining all the gathered data aiming to evaluate possible synergies in the information contained in both data sets before the model development. To normalize the weight of each variable in the PCA, variables were scaled and centered. Finally, the capability of the potentiometric E-tongue to discriminate olive oils (stored in the original bottles, which were left open or closed after each sampling) according to the simulated domestic consumption time period $(0,7,14,21$, and 28 days) was studied using LDA coupled with the metaheuristic simulated annealing (SA) variable selection algorithm (Bertsimas \& Tsitsiklis, 1993; Cadima, Cerdeira, \& Minhoto, 2004). E-tongue-LDA-SA models were established based on the best subsets of the 40 signal profiles generated during the electrochemical analysis, which were selected by the SA algorithm, aiming to minimize noise effects due to the inclusion of redundant potentiometric signals. The LDA predictive performance was evaluated using the leave-one-out cross-validation (LOO-CV) technique taking into account the dimension of the independent dataset, which, thus, not allows implementing a more robust $\mathrm{CV}$ technique or to use an independent test dataset. The classification performance of each LDA model was also graphically evaluated by plotting the main discriminant functions and the class membership boundary lines based on the posterior probabilities computed using the Bayes' theorem (which enables controlling over-fitting issues) (Bishop, 2006). All the statistical analyses were performed using the Subselect (Cadima et al., 2004; Kuhn \& Johnson, 2013) and MASS (Venables \& Ripley, 2002) packages of the open source statistical program $R$ (version 2.15.1), at a 5\% significance level.

\section{Results and Discussion}

\section{Physicochemical and Chemical Temporal Changes of Olive Oil during 1-Month Olive Oil House-Use and the Influence on the Quality-Grade Classification}

Olive oil from each bottle (seven bottles of the same olive oil brand and lot, three of them left open, and the other four closed after each use) was analyzed weekly during 1-month house-use simulation. The physicochemical parameters legally required for the assessment of quality (FA, PV, $K 232, K 270$, and $\Delta K$ ), the OS, and the TP were evaluated. Two additional olive oil bottles (same brand and lot) were kept closed during the 1-month trial, being only open and analyzed at day 28 . The mean values $( \pm \mathrm{SD})$ are given in Table 1. For bottles stored open or closed after each use, the PV and $K_{232}$ values linearly increased with the consumption time period $(+0.81 \leq R$-Pearson $\leq+0.92)$. In contrast, OS and TP linearly decreased with the time period $(-0.94 \leq R$-Pearson $\leq-0.83)$. These trends are in agreement with those reported in the literature regarding the shelf life of bottled olive oils during their storage along the commercial distribution chain (Jabeur et al., 2016; Rodrigues et al., 2016). Moreover, it is clear that the levels of all physicochemical and chemical parameters changed during the 1-month simulated olive oil domestic consumption (Table 1), with the time-evolution similar but slightly different depending on if the bottles remained open or were closed after each use. Furthermore, the two-way ANOVA showed that for FA, PV, and OS, no significant interaction effects were found ( $P$-value $\geq 0.1289$, for two-way ANOVA) and in contrast, for $K_{232}, K_{270}, \Delta K$, and TP significant interaction effects were found $(P$-value $\leq 0.0177$ for two-way ANOVA), for which disordinal effects were observed, requiring the interpretation of the results based on the plot trends of the estimated marginal means (Field, 2009). Taking into account this issue, it was decided to discuss all the results using the plots of the estimated marginal means (Fig. 1). Overall, the plots showed that the levels of FA were significantly influenced by how olive oil bottles were conditioned after each sample withdrawal (i.e., if after domestic-use simulation, bottles were closed or left open). Lower values were found for olive oils kept in open bottles, although no significant effect was observed due to the storage time period. Nevertheless, it should be noticed that, from a quality point of view, the observed variation (Fig. 1) was quite narrow along the 1-month trial (varying between $0.15 \%$ and $0.18 \%$ ). In contrast, PV were significantly affected by the storage time period whose levels increased with the storage time period and no significant effect was found due to the manner the bottles were conditioned after each simulated domestic use (i.e., closed or left open). A similar situation was shown for the OS; values significantly decreased with the storage time period but bottle storage type had no significant effect. Once again, it should be highlighted that the maximum OS decrease over the 1-month trial is around 1 hour, which is of the magnitude of the Rancimat method uncertainty; thus, from a practical point of view this finding must be used with caution. The $K_{232}$ values significantly increased with the storage period, with no significant effect observed due to the type of bottle storage. In contrast, the TP levels showed a significant 


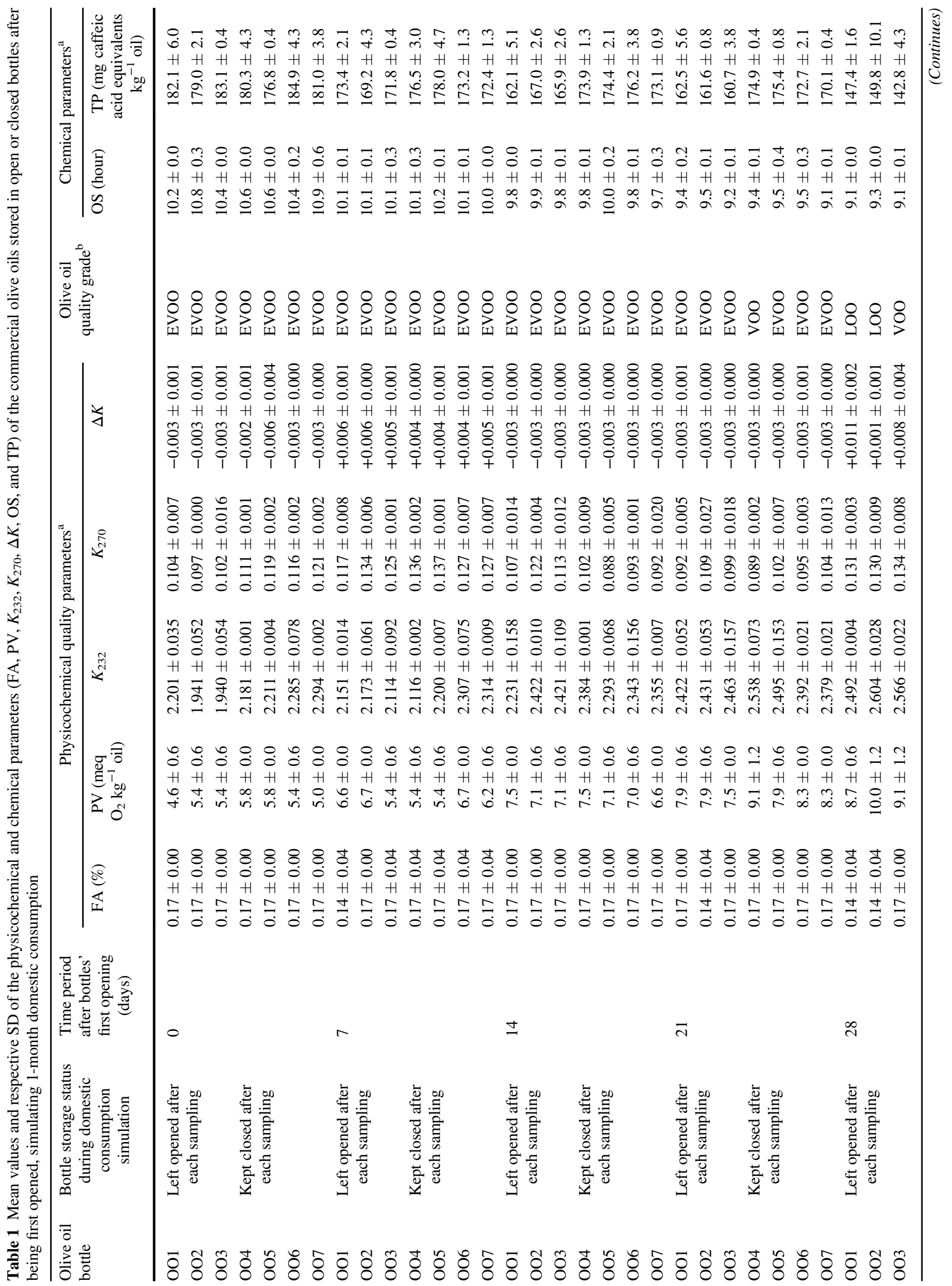




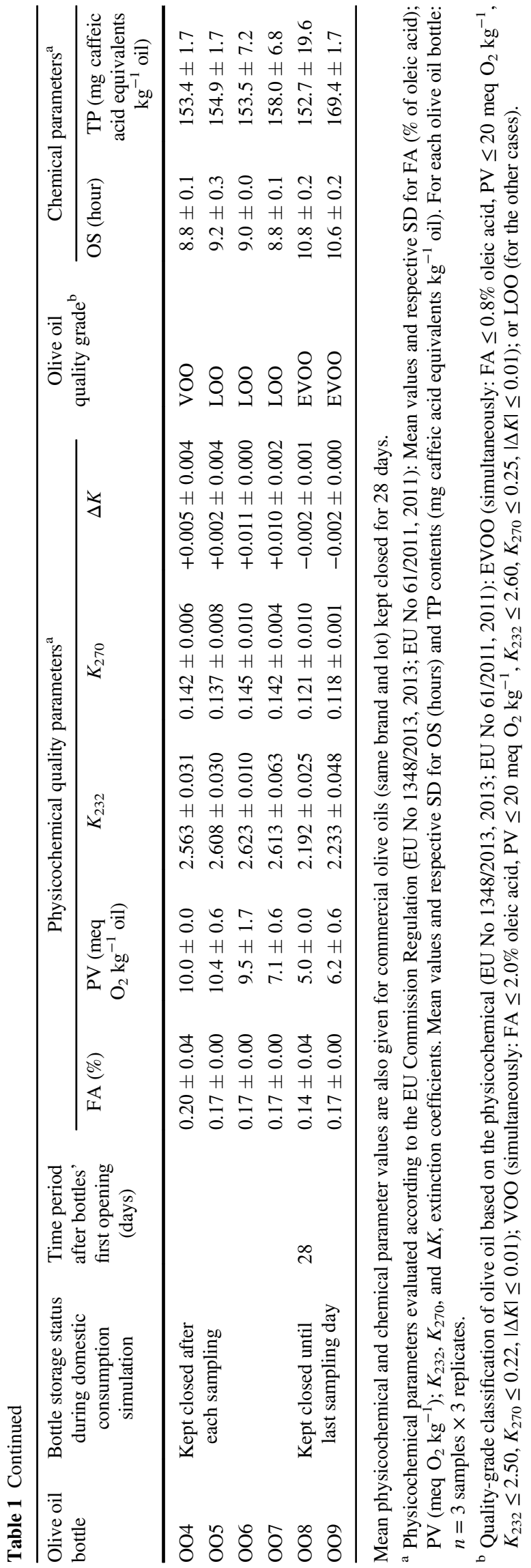

reduction with the storage time, with the decrease more drastic for olive oils stored in open bottles, which could be due to the volatile characteristics of the majority of the phenolic compounds. Regarding the two other parameters $\left(K_{270}\right.$ and $\left.\Delta K\right)$ although the main effects evaluated had a significant influence on their temporal evolution, no specific trend could be observed. Finally, based on the Student's $t$-test, it can be concluded that FA, PV, $K_{232}, K_{270}$, $\Delta K$, OS, and TP levels for the olive oils (same brand and lot) that were stored in the closed original bottles during 28 days (OO8 and OO9; Table 1) were not significantly different $(P$-value $\geq 0.0768)$ from those recorded for samples opened at day 0 (OO1-OO7). This finding indicates that the usual storage conditions of unopened olive oil bottles did not have a significant influence, per se, on the physicochemical levels for a 1-month time period. Similarly, it should be emphasized that the temporal level changes of the five physicochemical quality parameters (FA, PV, $K_{232}, K_{270}$, and $\Delta K$ ) did not alter the initial olive oils' high-quality grade (EVOO classification) during the first 21 days of simulated house use with the exception of olive oil OO4, which would be classified as VOO after this time period, due to the $K_{232}$ levels $\left(2.50<K_{232}<2.60\right)$. However, after 28 days, for five of the seven bottles stored open or closed (OO1, OO2, OO5, OO6, and OO7), the levels of $K_{232}$ and/or $\Delta K$ were greater than 2.60 or 0.01 , respectively, indicating an olive oil quality loss, leading to a LOO classification. The olive oils contained in the two other bottles (OO3 and $\mathrm{OO} 4)$ showed a lower quality degradation and so, could still be classified as VOO. It should be remarked that LOO cannot be sold commercially without being refined and thus, it is of major relevance to establish the maximum shelf life of an olive oil during usual house use to minimize the possibility of consuming lowquality olive oil. These results confirmed that, after the first olive oil withdrawal from each bottle, the physicochemical quality decreased in such an extent that after 28 days of simulated domestic use, the quality grade of olive oil could decrease from EVOO to VOO or even LOO. This information could be used by olive oil producers to advise consumers about the optimal consumption period after the first bottle opening (e.g., approximately 2-3 weeks), i.e., the time during which the initial quality grade of olive oil would remain (EVOO) or decrease to VOO, increasing the consumers' confidence and satisfaction when consuming this high-value food product. It should be remarked that, although the "best before" date of all olive oil bottles was not reached, the $K_{232}$ initial levels were slightly high (varying between $1.940 \pm 0.054$ and $2.294 \pm 0.002$ ), which, together with the fact that Arbequina olive oils were used, could partially explain the extent of the quality degradation observed (from EVOO to VOO or LOO), which could be lower if higher-quality 

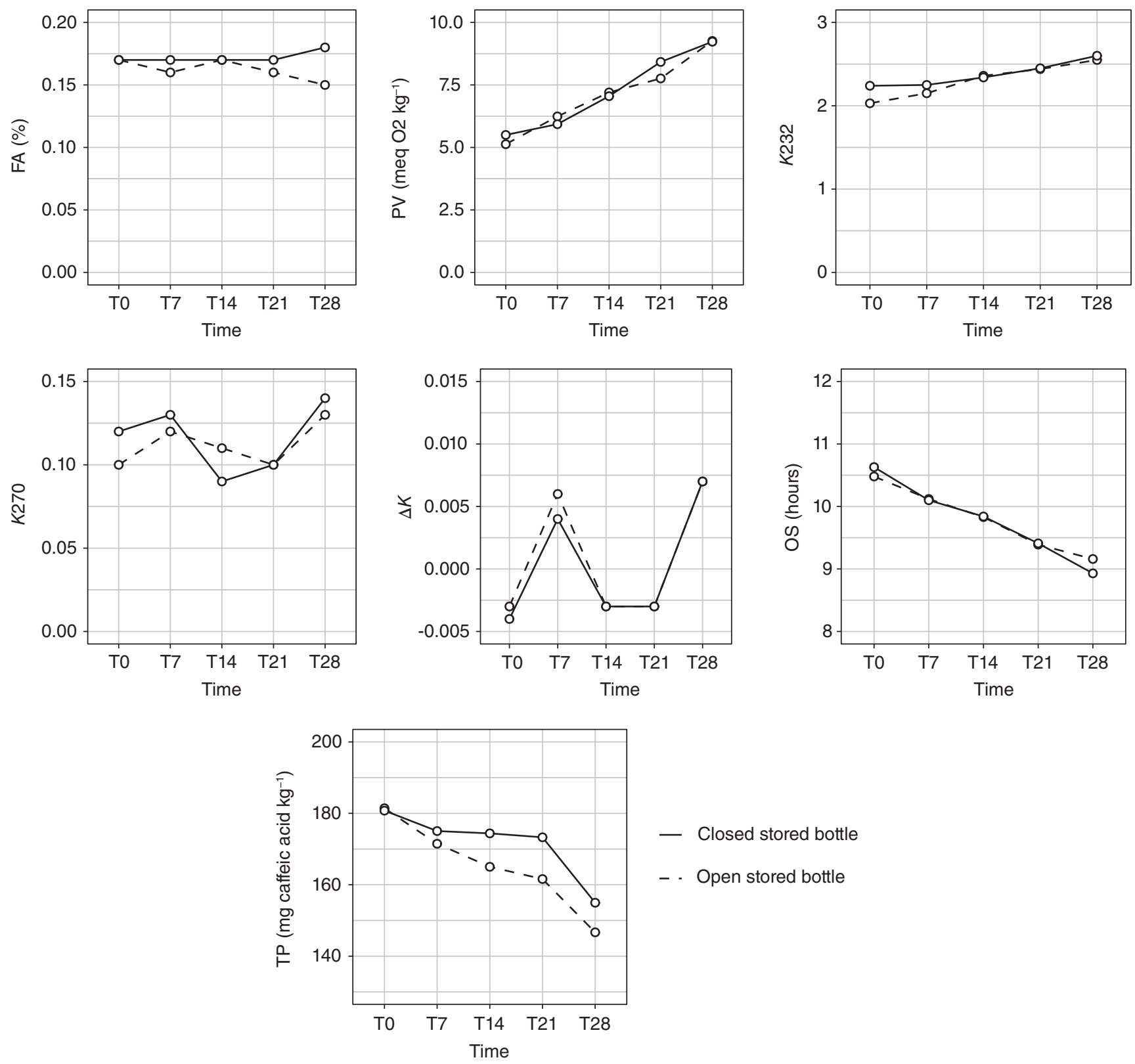

Fig. 1 Estimated margin means for physicochemical (FA, PV, $K_{232}, K_{270}$, and $\Delta K$ ) and chemical (OS and TP) parameters of olive oils during the analysis of bottled olive oils during 1-month simulated domestic consumption, which were stored in open or closed bottles after each withdrawal

olive oils from other olive cultivars (e.g., cvs Picual or Coratina) were studied.

\section{Temporal Evolution of the Olive Oil Sensory Profiles during a Typical 1-Month Olive Oil Domestic Consumption Period and Its Impact on the Legal Quality Classification}

Each olive oil bottle was assessed weekly by a trained sensory panel (in total nine bottles, two of them only being evaluated at the last sampling day). The mean intensities
$( \pm \mathrm{SD})$ of negative and positive olfactory and gustatoryretronasal sensations perceived are given in Tables $2-4$. It should be noticed that sensory attributes intensities lower than 2, although perceived by trained panelists are hardly detected by consumers. From these results, it can be observed that the intensity of olfactory and gustatoryretronasal sensations of olive ripe fruit and dry hay grass increases with the consumption time period $(+0.84 \leq R$ Pearson $\leq+0.94$ ), regardless of how the bottles are stored (left open or kept closed after each house use). It should be remarked that, the behavior observed for ripe-fruit 


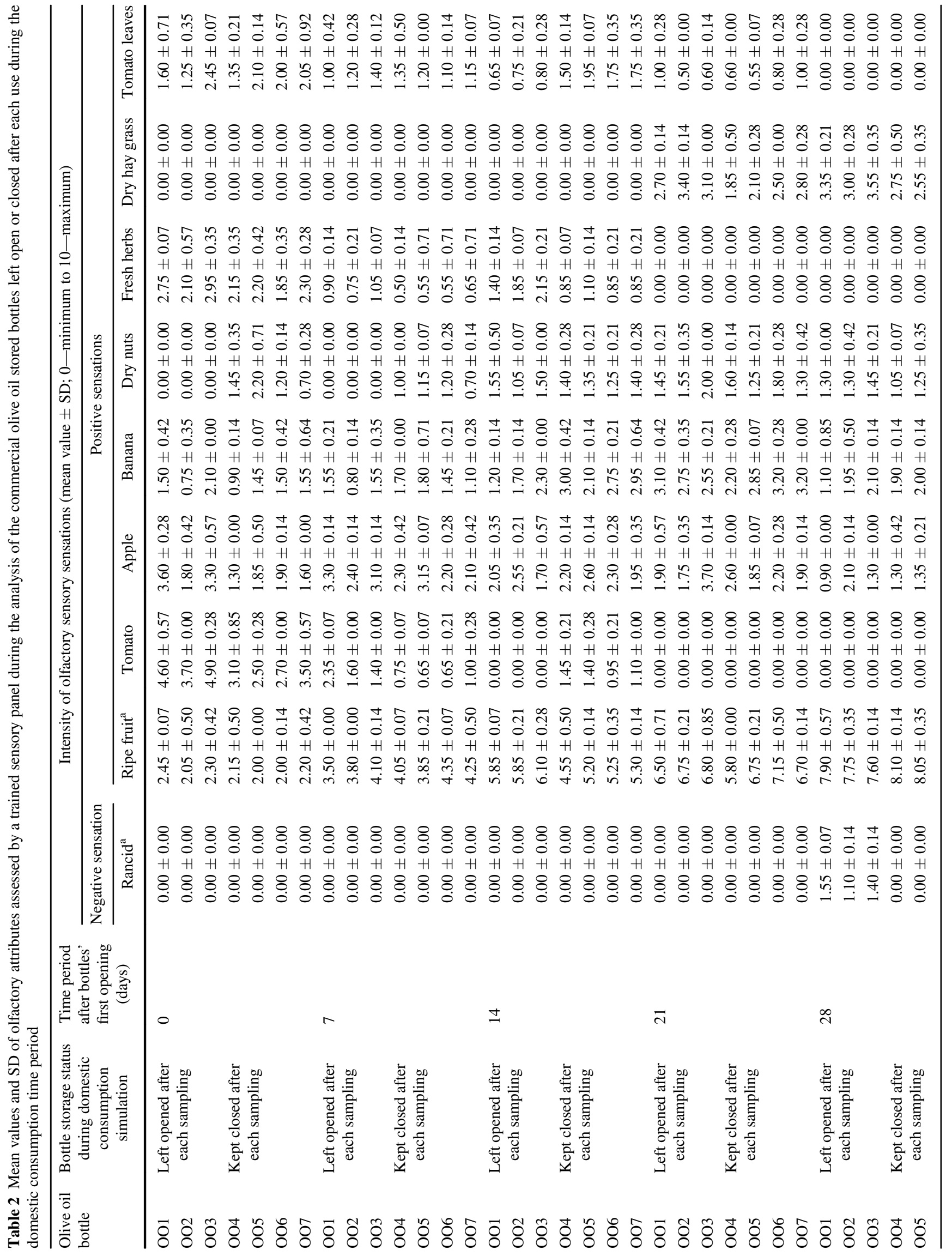




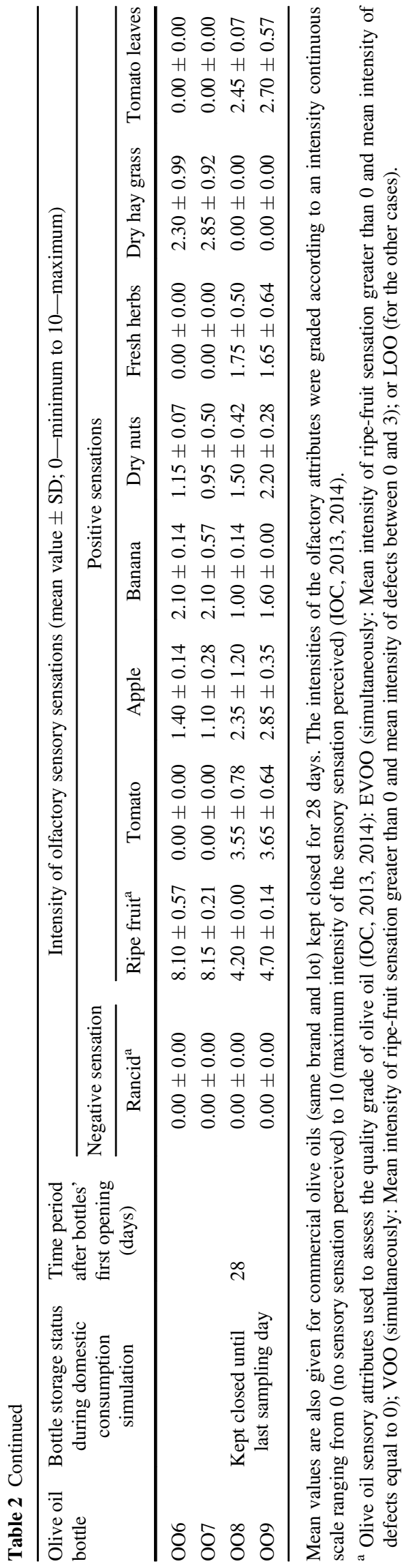

sensation has an opposite trend compared to the usual fruity attribute (which is mainly related to the fresh green sensation). In contrast, olfactory and gustatory-retronasal attributes (tomato, fresh herbs, and tomato leaves) as well as bitterness and pungent gustatory sensations showed a linear decrease trend with the olive oil consumption time period for bottles left open or kept closed after each domestic use $(-0.87 \leq R$-Pearson $\leq-0.77)$. Finally, although not so evident, it could also be tentatively stated that complexity and persistence of olive oil decrease during the simulation period $(-0.81 \leq R$-Pearson $\leq-0.72)$. The temporal changes of the sensory attributes were investigated by means of the two-way ANOVA. The analysis carried out showed that, for the negative and positive olfactory and gustatory-retronasal attributes of olive oil, significant interaction effects could be found $(P$-value $\leq 0.0437$ for all olfactory sensations; $P$-value $\leq 0.0500$ for gustatoryretronasal sensations with the exception of bitter, pungent dry nuts, and harmony, for which $P$-value $\geq 0.2018$ ). These findings together with the crossing lines observed for the majority of the sensory attributes perceived (Figs. 2 and 3) showed the nonadditive character of the significant interaction effects and then the need of a graphical interpretation of the results (Field, 2009). The plots of the estimated marginal means showed that during the first 21 days, no rancid sensation could be detected by the trained sensory panel in any bottle but not after 28 days because mean intensity values ranging from $1.10 \pm 0.14$ to $1.60 \pm 0.28$ were perceived during the evaluation (a decrease of the olive oil quality from EVOO to VOO). The graphical analysis also confirmed that the mean intensities of the fresh sensory notes (olfactory and gustatory-retronasal) like tomato, apple, fresh herbs, and tomato leave sensations decreased along the consumption time period. Simultaneously, olive oils kept in open bottles during the simulation showed higher intensity levels of the abovementioned attributes, compared to the olive oils stored in closed bottles, with the exception of tomato leaves' sensation. A similar decrease trend with the simulation time period was observed for bitterness and pungent olive oil intensities although no significant difference could be perceived between olive oils kept in open or closed bottles. An opposite trend with time (i.e., intensity perception increase with the simulation timeperiod) was observed for mature notes like ripe-fruit and dry hay grass sensations. The overall final complexity and persistence of the olive oils evaluated showed a clear decrease trend over time. The results of Student's $t$-tests pointed out that within the samples opened for the first time at day 0 or at day 28 , no significant statistical differences could be found for the evaluated negative- or positivesensory attributes $(P$-value $\geq 0.0560$, for Student's $t$-test with or without Welch's correction for unequal variances), except for ripe-fruit sensation $(P$-value $\leq 0.0015)$ and 


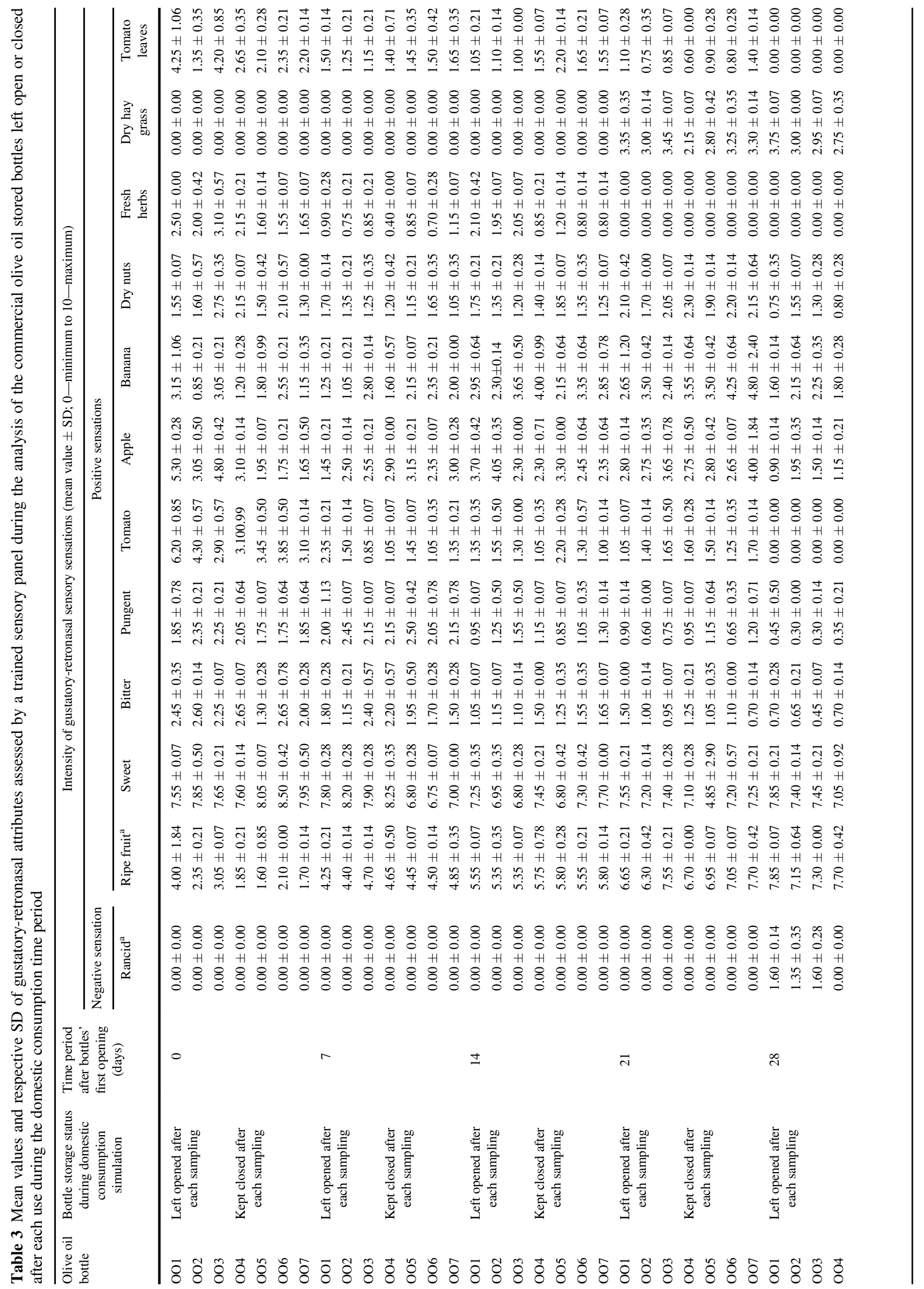


tomato leaves' olfactory sensation $(P$-value $=0.0253)$. It could be concluded that the temporal changes of the olive oils' sensory intensities were mainly due to the olive oil house use and not due to the usual storage conditions of the unopened olive oil bottles (1 month).

\section{PCA: Olive Oils' Unsupervised Grouping According to the Simulated Domestic-Use Time Period based on the Temporal Changes of the Physicochemical, Chemical, and Sensory Attributes}

The levels of physicochemical and chemical parameters as well as of the sensory attributes of EVOO changed during the 1-month simulation. The overall change in magnitude led in some cases to a drastic decrease in the quality grade of olive oil (from EVOO to VOO or even LOO), mainly after 28 days, which is of utmost relevance because LOO cannot be commercialized for human consumption unless it is previously refined. Therefore, the possibility of using the entire dataset (low level of the abstraction data fusion approach) to achieve a natural split of the olive oils according to the domestic consumption time period $(0,7,14,21$, and 28 days) or the type of bottle storage after first opening was evaluated using a PCA. The 2D plot of the first two PC (which explained $57.1 \%$ of the data variability) is shown in Fig. 4. It shows that olive oils are naturally grouped according to the increase of the time period of the olive oil consumption. Indeed, the alignment of the unsupervised formed groups along the first PC strengthens the finding that the physicochemical, chemical, and sensory properties of olive oil are mostly influenced by the consumption time period, and also by the bottle storage condition (open or closed). Furthermore, the olive oils stored in bottles that remained closed during the entire consumption time period (i.e., only open on the 28th day) were located near to those that were open at day 0 , confirming that the storage time period of unopened bottles did not significantly affect the overall characteristics of the olive oils during the month. Moreover, this procedure could be used to determine for how long an olive oil bottle has been open and used for domestic consumption, although it would require several time-consuming and costly assays (physicochemical, chemical, and sensory data).

\section{E-Tongue-LDA-SA Approach: Discrimination of Olive Oils According to the Consumption Time Period for Bottles Left Open or Closed After Each Simulated Domestic Use}

The results (Tables 1-4) showed that the quality grade of an olive oil decreases during the 1-month simulated house use due to the great increase of the $K_{232}$ and $\Delta K$ levels and due to the appearance of the rancid sensory defect. The 
Table 4 Mean values and SD of overall sensory (olfactory and gustatory-retronasal) intensities assessed by a trained sensory panel during the analysis of the commercial olive oil stored bottles left open or closed after each use during the domestic consumption time period

\begin{tabular}{|c|c|c|c|c|c|c|}
\hline \multirow{2}{*}{$\begin{array}{l}\text { Olive oil } \\
\text { bottle }\end{array}$} & \multirow{2}{*}{$\begin{array}{l}\text { Bottle storage status } \\
\text { during domestic } \\
\text { consumption } \\
\text { simulation }\end{array}$} & \multirow{2}{*}{$\begin{array}{l}\text { Time period } \\
\text { after bottles' } \\
\text { first opening } \\
\text { (days) }\end{array}$} & \multicolumn{3}{|c|}{ Overall positive sensation (olfactory and gustatory-retronasal sensations) } & \multirow[b]{2}{*}{ Persistence } \\
\hline & & & $\begin{array}{l}\text { Olfactory } \\
\text { harmony }\end{array}$ & $\begin{array}{l}\text { Gustatory-retronasal } \\
\text { harmony }\end{array}$ & Complexity & \\
\hline OO1 & \multirow{3}{*}{$\begin{array}{l}\text { Left opened after } \\
\text { each sampling }\end{array}$} & \multirow[t]{7}{*}{0} & $7.80 \pm 0.42$ & $7.75 \pm 0.35$ & $6.85 \pm 0.35$ & $6.85 \pm 0.92$ \\
\hline $\mathrm{OO} 2$ & & & $7.25 \pm 0.35$ & $7.25 \pm 0.35$ & $6.65 \pm 0.21$ & $6.85 \pm 0.07$ \\
\hline $\mathrm{OO} 3$ & & & $7.05 \pm 0.07$ & $7.00 \pm 0.28$ & $6.70 \pm 0.00$ & $7.35 \pm 0.07$ \\
\hline $\mathrm{OO} 4$ & \multirow{4}{*}{$\begin{array}{c}\text { Kept closed after } \\
\text { each sampling }\end{array}$} & & $7.70 \pm 0.57$ & $7.30 \pm 0.42$ & $6.65 \pm 0.21$ & $6.85 \pm 0.21$ \\
\hline 005 & & & $8.10 \pm 0.00$ & $7.75 \pm 0.21$ & $6.75 \pm 0.21$ & $7.45 \pm 0.64$ \\
\hline OO6 & & & $7.80 \pm 0.00$ & $7.70 \pm 0.42$ & $7.25 \pm 0.21$ & $7.60 \pm 0.28$ \\
\hline OO7 & & & $7.70 \pm 0.28$ & $6.90 \pm 1.27$ & $7.20 \pm 0.00$ & $8.10 \pm 0.85$ \\
\hline OO1 & \multirow{3}{*}{$\begin{array}{l}\text { Left opened after } \\
\text { each sampling }\end{array}$} & \multirow[t]{7}{*}{7} & $8.45 \pm 0.21$ & $7.65 \pm 0.07$ & $7.30 \pm 0.28$ & $7.30 \pm 0.42$ \\
\hline $\mathrm{OO} 2$ & & & $8.35 \pm 0.21$ & $7.80 \pm 0.28$ & $6.85 \pm 0.21$ & $7.00 \pm 0.00$ \\
\hline $\mathrm{OO} 3$ & & & $7.45 \pm 0.21$ & $7.40 \pm 0.42$ & $6.85 \pm 0.35$ & $7.30 \pm 0.42$ \\
\hline OO4 & \multirow{4}{*}{$\begin{array}{l}\text { Kept closed after } \\
\text { each sampling }\end{array}$} & & $7.85 \pm 0.35$ & $7.90 \pm 0.28$ & $7.00 \pm 0.28$ & $7.40 \pm 0.57$ \\
\hline OO5 & & & $7.95 \pm 0.35$ & $7.40 \pm 0.14$ & $7.05 \pm 0.07$ & $7.10 \pm 0.14$ \\
\hline OO6 & & & $8.10 \pm 0.28$ & $7.50 \pm 0.28$ & $6.90 \pm 0.14$ & $6.65 \pm 0.50$ \\
\hline OO7 & & & $7.55 \pm 0.50$ & $7.25 \pm 0.35$ & $6.80 \pm 0.14$ & $6.40 \pm 0.42$ \\
\hline OO1 & \multirow{3}{*}{$\begin{array}{l}\text { Left opened after } \\
\text { each sampling }\end{array}$} & \multirow[t]{7}{*}{14} & $7.65 \pm 0.50$ & $7.95 \pm 0.07$ & $6.90 \pm 0.42$ & $6.60 \pm 0.42$ \\
\hline $\mathrm{OO} 2$ & & & $7.50 \pm 0.14$ & $7.35 \pm 0.50$ & $6.40 \pm 0.28$ & $6.50 \pm 0.57$ \\
\hline $\mathrm{OO} 3$ & & & $7.75 \pm 0.35$ & $7.90 \pm 0.14$ & $6.30 \pm 0.42$ & $6.55 \pm 0.35$ \\
\hline OO4 & \multirow{4}{*}{$\begin{array}{l}\text { Kept closed after } \\
\text { each sampling }\end{array}$} & & $7.50 \pm 0.28$ & $7.80 \pm 0.28$ & $6.20 \pm 0.28$ & $6.40 \pm 0.14$ \\
\hline OO5 & & & $7.55 \pm 0.07$ & $7.65 \pm 0.21$ & $6.90 \pm 0.14$ & $6.95 \pm 0.07$ \\
\hline OO6 & & & $7.75 \pm 0.35$ & $7.85 \pm 0.50$ & $6.50 \pm 0.14$ & $6.40 \pm 0.14$ \\
\hline OO7 & & & $7.85 \pm 0.21$ & $7.75 \pm 0.07$ & $6.65 \pm 0.50$ & $6.65 \pm 0.50$ \\
\hline OO1 & \multirow{3}{*}{$\begin{array}{l}\text { Left opened after } \\
\text { each sampling }\end{array}$} & \multirow[t]{7}{*}{21} & $7.85 \pm 0.21$ & $7.30 \pm 0.28$ & $6.40 \pm 0.14$ & $6.35 \pm 0.21$ \\
\hline $\mathrm{OO} 2$ & & & $7.90 \pm 0.42$ & $7.30 \pm 0.00$ & $5.80 \pm 0.28$ & $5.75 \pm 0.21$ \\
\hline $\mathrm{OO} 3$ & & & $7.40 \pm 0.57$ & $8.00 \pm 0.42$ & $6.55 \pm 0.50$ & $6.40 \pm 0.42$ \\
\hline OO4 & \multirow{7}{*}{$\begin{array}{l}\text { Kept closed after } \\
\text { each sampling } \\
\text { Left opened after } \\
\text { each sampling }\end{array}$} & & $8.45 \pm 0.64$ & $7.95 \pm 0.07$ & $7.25 \pm 0.35$ & $7.25 \pm 0.50$ \\
\hline OO5 & & & $8.15 \pm 0.21$ & $7.70 \pm 0.28$ & $6.10 \pm 0.14$ & $6.45 \pm 0.35$ \\
\hline O06 & & & $8.10 \pm 0.14$ & $7.50 \pm 0.42$ & $6.15 \pm 0.35$ & $6.15 \pm 0.07$ \\
\hline OO7 & & & $8.25 \pm 0.07$ & $7.55 \pm 0.21$ & $7.10 \pm 0.28$ & $6.90 \pm 0.42$ \\
\hline OO1 & & 28 & $7.20 \pm 0.85$ & $7.65 \pm 0.50$ & $2.25 \pm 0.35$ & $5.75 \pm 0.78$ \\
\hline $\mathrm{OO} 2$ & & & $7.85 \pm 0.07$ & $7.35 \pm 0.50$ & $2.25 \pm 0.21$ & $5.45 \pm 0.07$ \\
\hline $\mathrm{OO} 3$ & & & $6.75 \pm 0.21$ & $7.30 \pm 0.42$ & $2.20 \pm 0.42$ & $5.45 \pm 0.21$ \\
\hline OO4 & \multirow{4}{*}{$\begin{array}{c}\text { Kept closed after } \\
\text { each sampling }\end{array}$} & & $6.90 \pm 0.00$ & $6.85 \pm 0.92$ & $4.45 \pm 0.35$ & $6.10 \pm 0.71$ \\
\hline OO5 & & & $6.85 \pm 0.07$ & $7.35 \pm 0.21$ & $4.30 \pm 0.42$ & $5.60 \pm 0.00$ \\
\hline OO6 & & & $6.85 \pm 0.07$ & $6.90 \pm 0.00$ & $4.65 \pm 0.35$ & $5.65 \pm 0.07$ \\
\hline OO7 & & & $6.35 \pm 0.35$ & $7.55 \pm 0.07$ & $4.15 \pm 0.35$ & $6.25 \pm 0.35$ \\
\hline OO8 & \multirow{2}{*}{$\begin{array}{l}\text { Kept closed until } \\
\text { last sampling day }\end{array}$} & \multirow[t]{2}{*}{28} & $8.00 \pm 0.14$ & $8.15 \pm 0.21$ & $7.70 \pm 0.28$ & $8.25 \pm 0.35$ \\
\hline OO9 & & & $8.15 \pm 0.21$ & $8.15 \pm 0.07$ & $7.30 \pm 0.42$ & $7.95 \pm 0.21$ \\
\hline
\end{tabular}

Mean values are also given for commercial olive oils (same brand and lot) kept closed for 28 days. The intensities of the overall sensory attributes were graded according to an intensity continuous scale ranging from 0 (no sensory sensation perceived) to 10 (maximum intensity of the sensory sensation perceived) (IOC, 2013, 2014).

extent of temporal changes of the olive oil quality observed was smooth during the first 14-21 days of house-use simulation where the olive oil could still hold the EVOO or VOO grade classification. However, after 28 days, the quality of the remaining olive oil decreased considerably and the classification grade changed to VOO (2) or LOO (5). Thus, the possibility of applying a fast, cost-effective, and reliable electrochemical-based sensor device, such as the E-tongue, to discriminate the olive oils according to the time period would be of practical usefulness. Therefore, for 

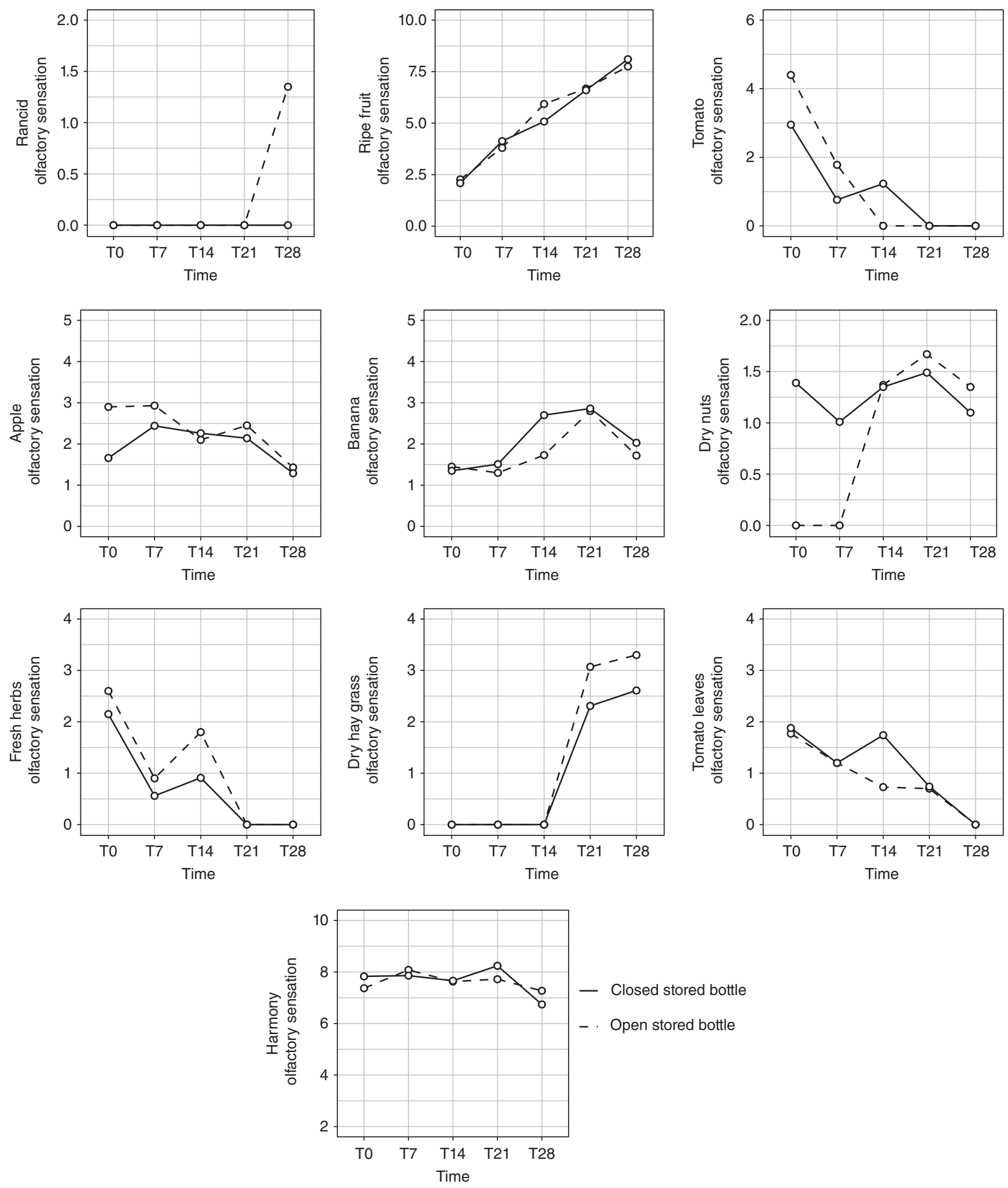

Fig. 2 Estimated marginal means of the intensities of negative- and positive-olfactory sensations perceived by a trained sensory panel during the analysis of bottled olive oils during 1-month simulated domestic consumption, which were stored in open or closed bottles after each withdrawal 

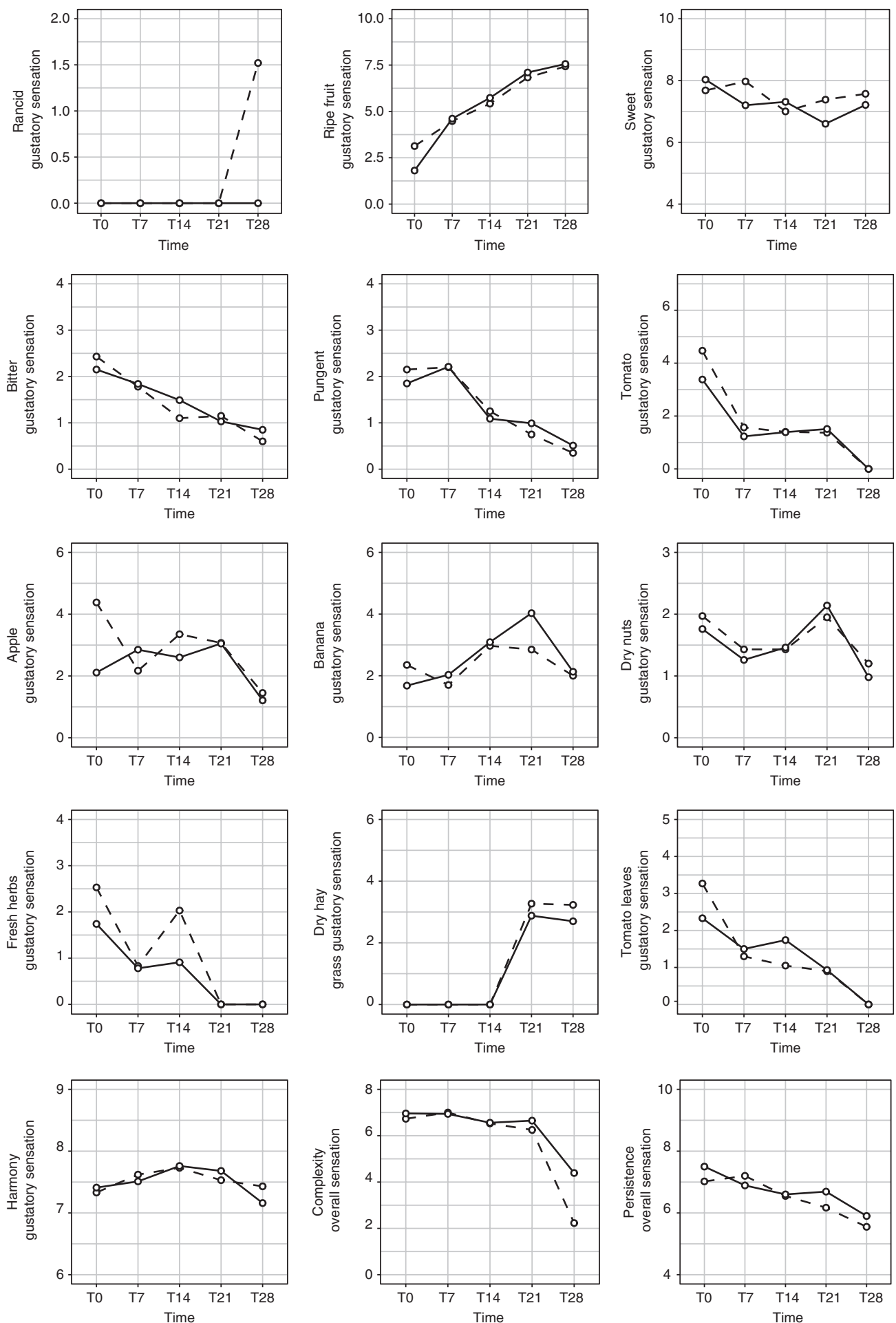

- Closed stored bottle

- Open stored bottle

Fig. 3 Estimated marginal means of the intensities of negative- and positive-gustatory-retronasal sensations and overall final complexity and persistence attributes perceived by a trained sensory panel during the analysis of bottled olive oils during 1 month simulated domestic consumption, which were stored in open or closed bottles after each withdrawal 


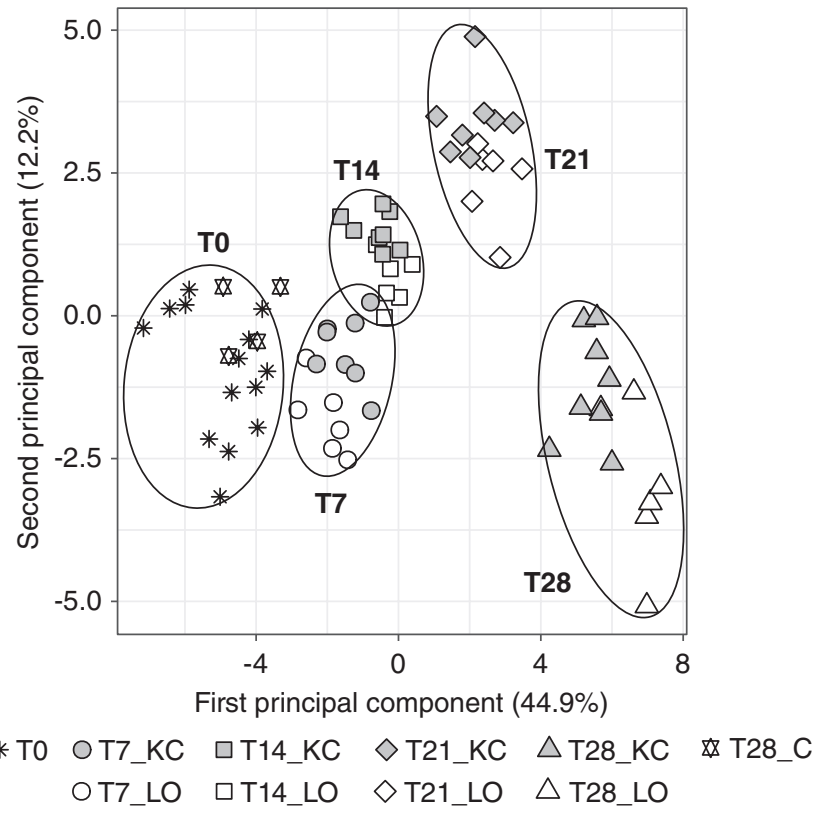

Fig. 4 PCA based on a low level of abstraction data fusion approach merging olive oils' physicochemical, chemical, and sensory experimental data. Symbol “*”: Bottles opened for the first time at day 0 (T0). Open circle symbols: Bottles kept open after each simulated domestic use at 7, 14, 21, and 28 days (T7_LO, T14_LO, T21_LO, and T28_LO, respectively). Filled square symbols: Bottles closed after each simulated domestic use at 7, 14, 21, and 28 days (T7_KC, T14_KC, T21_KC, and T28_KC, respectively). Symbol “œ”: Bottles that remained closed during the entire simulated domestic use, being only open for the first time after 28 days (T28_C)

the olive oils stored in the original bottles that were left open or closed after each simulated domestic use, E-tongue-LDA-SA models were established. For the olive

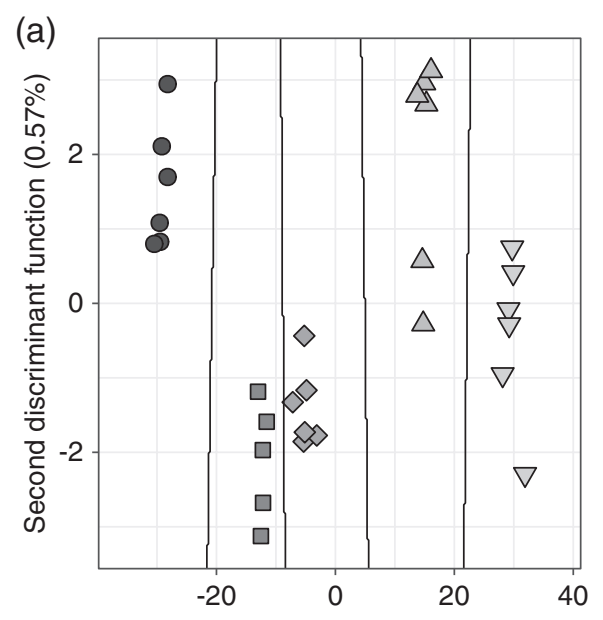

First discriminant function $(99.17 \%)$ oils stored in open bottles during the consumption time period, the multivariate classification model had three discriminant functions (explaining $99.2 \%, 0.6 \%$, and $0.2 \%$ of the data variability, respectively) and used the potentiometric information recorded by 15 sensor membranes (first array: S1:3, S1:6, S1:14, and S1:20; second array: S2:1, $\mathrm{S} 2: 3, \mathrm{~S} 2: 7, \mathrm{~S} 2: 9, \mathrm{~S} 2: 11-\mathrm{S} 2: 13, \mathrm{~S} 2: 16-\mathrm{S} 2: 18$, and $\mathrm{S} 2: 20$ ), allowing to correctly classify $100 \%$ (original grouped data) and $97 \%$ (LOO-CV) of the olive oil samples according to the storage time period $(0,7,14,21$, or 28 days) (Fig. 5a). Similarly, for the olive oils stored in the original bottles, which were closed after each use, the selected E-tongueLDA-SA model had three discriminant functions (explaining, $96.7 \%, 2.1 \%$, and $0.8 \%$ of the data variability) enabling to successfully discriminate the olive oils according to the consumption time period $(100 \%$ and $95 \%$ for original grouped data; Fig. 5b; and LOO-CV, respectively). The multivariate classification model was based on a higher number of sensor membranes (24 sensors-first array: $\mathrm{S} 1: 1-\mathrm{S} 1: 3, \mathrm{~S} 1: 5, \mathrm{~S} 1: 7, \mathrm{~S} 1: 8, \mathrm{~S} 1: 13, \mathrm{~S} 1: 16, \mathrm{~S} 1: 18, \mathrm{~S} 1: 20$, and $\mathrm{S} 1: 20$; second array: S2:1-S2:6, S2:8, S2:10, S2:12, $\mathrm{S} 2: 14, \mathrm{~S} 2: 16$, and S2:18-S2:20), probably due to the lower extent changes of physicochemical and sensory parameters observed for closed bottles compared to those left open during the simulation. The overall satisfactory classification performance of the E-tongue-LDA-SA approach is in accordance with previous studies that demonstrated the capability of this type of taste sensor device for olive oil analysis, including the assessment of levels of physicochemical quality parameters during 1 year of distribution storage (Rodrigues et al., 2016) as well as the evaluation of the intensities of positive- or negative-sensory attributes of

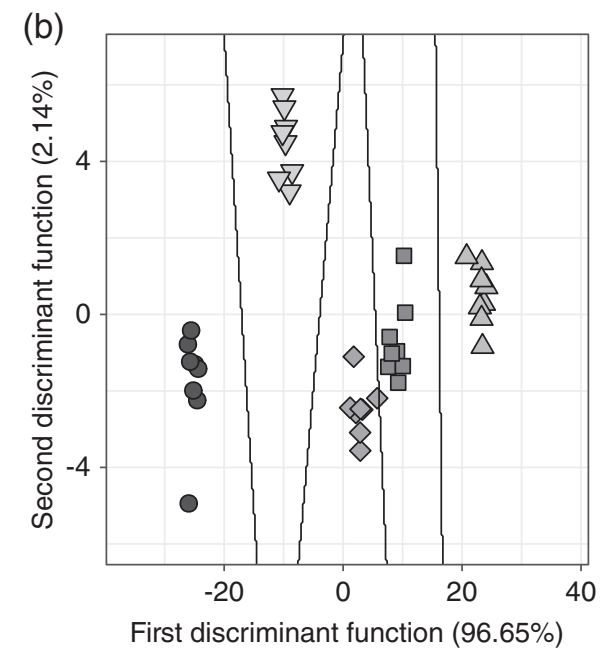

○ T0 $\square$ T7 $\diamond \mathrm{T} 14 \triangle \mathrm{T} 21 \nabla \mathrm{T} 28$

Fig. 5 Discrimination of olive oils during the simulated domestic consumption time period (T0, T7, T14, T21, and T28 for 0, 7, 14, 21, and 28 days, respectively) using the selected E-tongue-LDA-SA models. (a) Olive oils stored in the original bottles, which were left open after each simulated domestic use after being first opened. (b) Olive oils stored in the original bottles, which were closed after each simulated domestic use 
olive oils (Harzalli et al., 2018; Veloso et al., 2016, 2018). Finally, this electrochemical approach could be used as a single, fast, and low-cost tool to assess for how long an olive oil bottle has been open and used for domestic usage purposes, which as previously discussed could be directly correlated with the overall quality of the remained unused bottled olive oil.

\section{Conclusions}

This study confirmed that under the usual house-use consumption conditions (time period, temperature, and light exposure) the olive oils show significant changes in the levels of physicochemical, chemical, and sensory attributes, leading to an overall decrease of their quality grade. The physicochemical quality degradation and the appearance of rancid negative-sensory sensation contributed to the quality reduction of olive oil. Indeed, after 28 days of house-use simulation, the remaining olive oil stored in open bottles after each use was graded as LOO, which would prevent its commercialization, because the legal maximum levels would be exceeded. This finding strengthens the need for the shelf life assessment of olive oil for usual home-use conditions, allowing olive oil producers to guarantee the olive oil quality during predetermined domestic-use time periods (e.g., 2-3 weeks, after an olive oil bottle has been opened for the first time). Finally, the E-tongue device could be a fast and low-cost tool to determine for how long an olive oil bottle has been domestically consumed, as well as indirectly the optimal house-use shelf-life. This may allow producers to recommend consumers about the time period for domestic usage during which the desired olive oil quality is retained.

Acknowledgments This study was financially supported by Project POCI-01-0145-FEDER-006984-Associate Laboratory LSRE-LCM, Project UID/BIO/04469/2013 - CEB, and strategic project PEst-OE/ AGR/UI0690/2014 - CIMO all funded by FEDER-Fundo Europeu de Desenvolvimento Regional through COMPETE2020_-Programa Operacional Competitividade e Internacionalização (POCI), and by national funds through FCT-Fundação para a Ciência e a Tecnologia, Portugal. Nuno Rodrigues thanks FCT, POPH-QREN, and FSE for the Ph.D. grant (SFRH/BD/104038/2014). Souheib Oueslati is also grateful for the support from the Tunisian Ministry of Agriculture.

Conflict of interest The authors declare that they have no conflicts of interest.

\section{References}

Ayyad, Z., Valli, E., Bendini, A., Adrover-Obrador, S., Femenia, A., \& Toschi, T. G. (2015) Extra-virgin olive oil stored in different conditions: Focus on diglycerides. Italian Journal of Food Science, 27:166-172.

Bertsimas, D., \& Tsitsiklis, J. (1993) Simulated annealing. Statistical Science, 8:10-15.

Bishop, C. M. (2006) Pattern recognition and machine learning (1st ed.). New York, NY: Springer.

Cadima, J., Cerdeira, J. O., \& Minhoto, M. (2004) Computational aspects of algorithms for variable selection in the context of principal components. Computational Statistics \& Data Analysis, 47: 225-236.

Capannesi, C., Palchetti, I., Mascini, M., \& Parenti, A. (2000) Electrochemical sensor and biosensor for polyphenols detection in olive oils. Food Chemistry, 71:553-562.

Di Lecce, G., Bendini, A., Cerretani, L., Bonoli-Carbognin, M., \& Lercker, G. (2006) Evaluation of "home consumption conditions" on shelf-life of extra virgin olive oils. Industrie Alimentari, 45: 873-880.

EU No 1348/2013 (2013) Commission implementing regulation of 16 December 2013 amending Regulation No 2568/91/EEC on the characteristics of olive oil and olive-residue oil and on the relevant methods of analysis. Official Journal of the European Union, L338:31-67.

EU No 61/2011 (2011) Commission regulation of 24 January 2011 amending Regulation No 2568/91/EEC on the characteristics of olive oil and olive pomace oil and on the relevant methods of analysis. Official Journal of the European Union, L23:1-13.

Field, A. P. (2009) Discovering statistics using SPSS (3rd ed.). London: Sage.

Genovese, A., Caporaso, N., \& Sacchi, R. (2015) Temporal changes of virgin olive oil volatile compounds in a model system simulating domestic consumption: The role of biophenols. Food Research International, 77:670-674.

Harzalli, U., Rodrigues, N., Veloso, A. C. A., Dias, L. G., Pereira, J. A., Oueslati, S., \& Peres, A. M. (2018) A taste sensor device for unmasking admixing of rancid or winey-vinegary olive oil to extra virgin olive oil. Computers and Electronics in Agriculture, 144:222-231.

International Olive Council. (2013, November 6) Sensory analysis of olive oil-Method for the organoleptic assessment of virgin olive oil. COI/T.20/Doc. No 15/Rev, 18 pp. Retrieved from http://www. internationaloliveoil.org/

International Olive Council. (2014, June 17) IOC Mario Solinas quality award-Rules of the international competition for extra virgin olive oils. T.30/Doc. No, 9 pp. Retrieved from http://www. internationaloliveoil.org/

Jabeur, H., Zribi, A., \& Bouaziz, M. (2016) Extra-virgin olive oil and cheap vegetable oils: Distinction and detection of adulteration as determined by GC and chemometrics. Food Analytical Methods, 9: $712-723$.

Kobayashi, Y., Habara, M., Ikezazki, H., Chen, R., Naito, Y., \& Toko, K. (2010) Advanced taste sensors based on artificial lipids with global selectivity to basic taste qualities and high correlation to sensory scores. Sensors, 10:3411-3443.

Kuhn, M., \& Johnson, K. (2013) Applied predictive modeling. New York, NY: Springer Science Business Media.

Rodrigues, N., Dias, L. G., Veloso, A. C. A., Pereira, J. A., \& Peres, A. M. (2016) Monitoring olive oils quality and oxidative resistance during storage using an electronic tongue. $L W T-F o o d$ Science and Technology, 73:683-692.

Salek, R. N., Burešová, I., Kráčmar, S., Lorencová, E., Zálešáková, L., \& Dabash, V. (2017) Evaluation of selected physicochemical parameters of extra virgin olive oil commercialized in the Czech market and stored during a period of 5 months. Potravinarstvo Slovak Journal of Food Sciences, 11:664-672.

Sanaeifar, A., Jafari, A., \& Golmakani, M.-T. (2018) Fusion of dielectric spectroscopy and computer vision for quality characterization 
of olive oil during storage. Computers and Electronics in Agriculture, 145:142-152.

Spatari, C., De Luca, M., Ioele, G., \& Ragno, G. (2017) A critical evaluation of the analytical techniques in the photodegradation monitoring of edible oils. $L W T$-Food Science and Technology, 76:147-155.

Veloso, A. C. A., Dias, L. G., Rodrigues, N., Pereira, J. A., \& Peres, A. M. (2016) Sensory intensity assessment of olive oils using an electronic tongue. Talanta, 146:585-593.
Veloso, A. C. A., Silva, L. M., Rodrigues, N., Rebello, L. P. G., Dias, L. G., Pereira, J. A., \& Peres, A. M. (2018) Perception of olive oils sensory defects using a potentiometric taste device. Talanta, 176:610-618.

Venables, W. N., \& Ripley, B. D. (2002) Modern applied statistics with $S$ (statistics and computing) (4th ed.). New York, NY: Springer. 